\title{
First Asymmetric Synthesis of trans-3,4-Dimethyl-4-Arylpiperidines
}

\author{
Daniel P. Furkert* and Stephen M. Husbands \\ Department of Pharmacy \& Pharmacology, University of Bath, \\ Claverton Down, Bath, BA2 7AY, UK. \\ df218@bath.ac.uk and S.M.Husbands@bath.ac.uk
}

\section{Supporting Information}

\section{Contents}

S1-S5....... Experimental procedures and data

S6-S27. ${ }^{1} \mathrm{H}$ and ${ }^{13} \mathrm{C}$ NMR spectra, HPLC traces for $\mathbf{1 1}$ and $\mathbf{1}$

\section{General}

Chiral analytical HPLC was performed using an Astec Chirobiotic V2 vancomycin column with the indicated solvents. Signals in some ${ }^{1} \mathrm{H}$ NMR spectra appear broad due to conformational mobility of the $\mathrm{N}$-tert-butylsulfonyl piperidine system.

\section{Experimental}

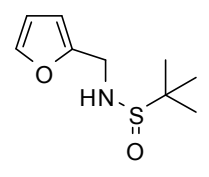

7

Compound 7: To a solution of furfurylamine $(1.15 \mathrm{~mL}, 12.4 \mathrm{mmol})$ and triethylamine $(5.0 \mathrm{~mL}$, $35.9 \mathrm{mmol})$ in dichloromethane $(20 \mathrm{~mL})$ at $0^{\circ} \mathrm{C}$ was added tert-butylsulfinyl chloride $(1.5 \mathrm{~mL}$, $12.1 \mathrm{mmol}$ ). The resultant slurry was stirred for $1 \mathrm{~h}$ then partitioned between dichloromethane and water. The organic phase was washed with water, brine, dried over magnesium sulfate and concentrated in vacuo. Column chromatography (50\% ethyl acetate/hexane) afforded sulfinimide 7 as a yellow oil $(2.17 \mathrm{~g}, 91 \%) . R_{f}=0.20$ (50\% ethyl acetate/hexane); IR (neat) 3433(br), 3207(br), 2959, 1641 (w), 1475, 1365, 1149, 1054(s), $1013 \mathrm{~cm}^{-1} ;{ }^{1} \mathrm{H}$ NMR (400 MHz, $\left.\mathrm{CDCl}_{3}\right) \delta 1.19(\mathrm{~s}, 9 \mathrm{H}), 3.47(\mathrm{brt}, J=5.6 \mathrm{~Hz}, 1 \mathrm{H}), 4.26(\mathrm{qd}, J=29.9,14.9$ and $5.2 \mathrm{~Hz}, 2 \mathrm{H})$, $6.24(\mathrm{dd}, J=3.2$ and $0.7 \mathrm{~Hz}, 1 \mathrm{H}), 6.31(\mathrm{dd}, J=3.2$ and $1.7 \mathrm{~Hz}, 1 \mathrm{H}), 7.35(\mathrm{dd}, J=1.7$ and $0.7 \mathrm{~Hz}, 1 \mathrm{H}) ;{ }^{13} \mathrm{C}$ NMR $\left(100 \mathrm{MHz}, \mathrm{CDCl}_{3}\right) \delta 22.5,42.2,56.0,107.8,110.3,142.4,151.8$; ms (ES) $\mathrm{m} / \mathrm{z}(\%) 224(\mathrm{M}+\mathrm{Na}, 39), 202\left(\mathrm{MH}^{+}, 100\right)$; High resolution ms: expected for $\mathrm{C}_{9} \mathrm{H}_{15} \mathrm{NNaO}_{2} \mathrm{~S}$ 224.0716, found 224.0706.

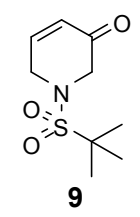

Compound 9: CAUTION: Crude compound 8 has been observed to decompose explosively if evaporated to dryness! To a solution of $7(2.0 \mathrm{~g}, 9.95 \mathrm{mmol})$ in dichloromethane $(100 \mathrm{~mL})$ was added $m$-CPBA ( $5.0 \mathrm{~g}, \leq 77 \%, 22.3 \mathrm{mmol})$. The reaction was stirred at rt until complete by TLC (ca. $3 \mathrm{~h}$ ) then diluted with dichloromethane, washed with saturated aqueous sodium bicarbonate $(2 \times)$, water and brine. The organic phase was dried over magnesium sulfate and carefully concentrated in vacuo to a volume of approximately $100 \mathrm{~mL}$ then cooled to $0^{\circ} \mathrm{C}$ and 
treated with triethylsilane $(1.60 \mathrm{~mL}, 10.0 \mathrm{mmol})$ and $\mathrm{BF}_{3} \cdot \mathrm{OEt}_{2}(1.23 \mathrm{~mL}, 9.95 \mathrm{mmol})$. After $3 \mathrm{~h}$ the reaction was diluted with dichloromethane, washed with sodium bicarbonate-sodium sulfite (1:1) sodium bicarbonate, water and brine. The organic phase was dried over magnesium sulfate and concentrated in vacuo to afford a crystalline solid $(1.95 \mathrm{~g})$. The crude material was taken up in minimum ethyl acetate and triturated with hexane to yield $9(1.68 \mathrm{~g}$, $78 \%)$ as colourless crystals. $R_{f}=0.24\left(50 \%\right.$ ethyl acetate/hexane); $\mathrm{mp} 113-114^{\circ} \mathrm{C}$; $\mathrm{IR}(\mathrm{KBr})$ 2976, 1684, 1300, $1118 \mathrm{~cm}^{-1} ;{ }^{1} \mathrm{H}$ NMR $\left(400 \mathrm{MHz}, \mathrm{CDCl}_{3}\right) \delta 1.33(\mathrm{~s}, 9 \mathrm{H}), 4.00(\mathrm{~s}, 2 \mathrm{H}), 4.17(\mathrm{~s}$, $2 \mathrm{H}$ ), 6.19 (ddd, $J=10.4,4.2$ and $2.2 \mathrm{~Hz}, 1 \mathrm{H}$ ), 7.05 (ddd, $J=10.4,3.6$ and $3.6 \mathrm{~Hz}, 1 \mathrm{H}$ ); ${ }^{13} \mathrm{C} \mathrm{NMR}\left(100 \mathrm{MHz}, \mathrm{CDCl}_{3}\right) \delta 24.4,46.2,54.4,62.1,128.3,147.8,192.7 ; \mathrm{ms}(\mathrm{ES}+) \mathrm{m} / \mathrm{z}(\%)$ $240\left(\mathrm{M}^{+}+\mathrm{Na}, 100\right)$; High resolution ms: expected for $\mathrm{C}_{9} \mathrm{H}_{15} \mathrm{NNaO}_{3} \mathrm{~S} 240.0665$, found 240.0663 .

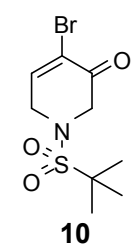

Compound 10: A solution of bromine $(144 \mu \mathrm{L}, 2.82 \mathrm{mmol})$ in dichloromethane $(10 \mathrm{~mL})$ was added dropwise to a solution of enone $9(0.60 \mathrm{~g}, 2.76 \mathrm{mmol})$ in dichloromethane $(30 \mathrm{~mL})$ at $0^{\circ} \mathrm{C}$. After $30 \mathrm{~min}$ triethylamine $(0.40 \mathrm{~mL}, 2.87 \mathrm{mmol}$ ) was added (some fuming) and stirring continued at $0^{\circ} \mathrm{C}$ for a further $30 \mathrm{~min}$. The reaction mixture was then diluted with dichloromethane, washed with water $(2 \times)$, saturated aqueous sodium bicarbonate and brine, dried over magnesium sulfate and concentrated in vacuo to give an orange crystalline solid $(0.79 \mathrm{~g})$. Recrystallisation from methanol afforded $10(0.73 \mathrm{~g}, 89 \%)$ as colourless needles. $\mathrm{R}_{f}=0.36$ (50\% ethyl acetate/hexane); $\mathrm{mp} 128-130^{\circ} \mathrm{C}$; IR (KBr) 2927, 1697, 1690, 1327, 1306, $1133 \mathrm{~cm}^{-1} ;{ }^{1} \mathrm{H}$ NMR $\left(400 \mathrm{MHz}, \mathrm{CDCl}_{3}\right) \delta 1.35(\mathrm{~s}, 9 \mathrm{H}), 4.19(\mathrm{~s}, 2 \mathrm{H}), 4.23(\mathrm{~d}, \mathrm{~J}=3.9 \mathrm{~Hz}, 2 \mathrm{H})$, $7.44(\mathrm{t}, \mathrm{J}=3.9 \mathrm{~Hz}, 1 \mathrm{H}) ;{ }^{13} \mathrm{C}$ NMR $\left(100 \mathrm{MHz}, \mathrm{CDCl}_{3}\right) \delta 24.2,48.1,54.6,62.1,122.0,146.9$, 185.0; ms (ES) $\mathrm{m} / \mathrm{z}(\%) 320(100)$, 318(100), 270(27), 178(12), 176(11); High resolution ms: expected for $\mathrm{C}_{9} \mathrm{H}_{14} \mathrm{NNaO}_{3} \mathrm{~S} 317.9770$, found 317.9762 .

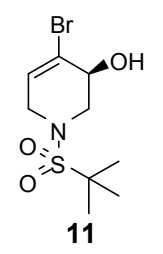

Compound 11: Racemic: To a solution of recrystallised $10(0.53 \mathrm{~g}, 1.78 \mathrm{mmol})$ and $\mathrm{CeCl}_{3} \cdot 7 \mathrm{H}_{2} \mathrm{O}(0.69 \mathrm{~g}, 1.87 \mathrm{mmol})$ in methanol-THF $(1: 1,20 \mathrm{~mL})$ at $0^{\circ} \mathrm{C}$ was added sodium borohydride $(71 \mathrm{mg}, 1.87 \mathrm{mmol})$. After $15 \mathrm{~min}$ the reaction was quenched by the addition of water then partitioned between dichloromethane and water. The organic phase was washed with water, brine, dried over magnesium sulfate and concentrated in vacuo. Column chromatography (25\% ethyl acetate/hexane) afforded rac-11 as a colourless crystalline solid $(0.51 \mathrm{~g}, 96 \%)$. Enantioselective: A solution of $(+)-(R)$-diphenylprolinol $(70 \mathrm{mg}, 0.28 \mathrm{mmol})$ and methylboronic acid $(16.6 \mathrm{mg}, 0.28 \mathrm{mmol})$ in anhydrous toluene containing activated $4 \AA$ molecular sieves was stirred for $60 \mathrm{~min}$ at rt. Filtration and concentration in vacuo afforded a colourless foam $(80 \mathrm{mg})$ which was taken up in THF $(8 \mathrm{~mL})$ and cooled to $0^{\circ} \mathrm{C}$. Borane- $N, N-$ diethylaniline complex $(0.25 \mathrm{~mL}, 1.41 \mathrm{mmol})$ was then added followed by a solution of $\mathbf{1 0}$ $(0.45 \mathrm{~g}, 1.51 \mathrm{mmol})$ in THF $(15 \mathrm{~mL})$ dropwise via cannula over $10 \mathrm{~min}$. The reaction mixture was stirred for $16 \mathrm{~h}$ warming slowly to rt before excess borane was quenched with methanol $(1 \mathrm{~mL})$ and the solvents removed. The residue was taken up in dichloromethane and the organic phase washed with $1 \mathrm{M} \mathrm{HCl}(2 \times)$, water, brine, dried over magnesium sulfate and concentrated in vacuo. Column chromatography $(20-40 \%$ ethyl acetate/hexane) afforded $(-)-(S)-11$ as a colourless oil that crystallised upon storage in the freezer $(0.40 \mathrm{~g}, 88 \%)$. $\mathrm{R}_{f}=0.5\left(50 \%\right.$ ethyl acetate/hexane); $[\alpha]_{\mathrm{D}}-46.0\left(\mathrm{CHCl}_{3}, c \quad 0.052\right) ; \mathrm{mp} 97-99^{\circ} \mathrm{C}$; IR (neat) 3481(br), 2980, 2917, 1312(s), 1131, 1067; ${ }^{1} \mathrm{H}$ NMR (400 MHz, $\left.\mathrm{CDCl}_{3}\right) \delta 1.37$ (s, 9H), 2.83 (br s. $1 \mathrm{H}), 3.63(\mathrm{~d}, J=5.6 \mathrm{~Hz}, 2 \mathrm{H}), 3.90(\mathrm{q}, 2 \mathrm{H}), 4.18(\mathrm{br} \mathrm{s}, 1 \mathrm{H}), 6.21(\mathrm{t}, J=3.5 \mathrm{~Hz}) ;{ }^{13} \mathrm{C}$ NMR 
$\left(100 \mathrm{MHz}, \mathrm{CDCl}_{3}\right) \delta 24.5,47.9, \quad 51.6,62.0,68.6,124.1,128.8 ; \mathrm{ms}(\mathrm{ES}) \mathrm{m} / \mathrm{z}(\%)$ $319\left({ }^{79} \mathrm{Br}^{2} \mathrm{M}^{+}+\mathrm{Na}, 87\right), 321\left(\left[{ }^{81} \mathrm{Br}\right] \mathrm{M}^{+}+\mathrm{Na}, 100\right)$; High resolution ms: expected for $\mathrm{C}_{9} \mathrm{H}_{17} \mathrm{BrNO}_{3} \mathrm{~S}$ 298.0107, found 298.0096. HPLC: $t_{R}($ minor $)=13.9 \mathrm{~min}, t_{\mathrm{R}}($ major $)=15.6 \mathrm{~min}$ detected at 203 $\mathrm{nm}, 97: 13$ (hexane-EtOH), $1 \mathrm{~mL} / \mathrm{min}$.<smiles>[Y2]C(C)(C)S(=O)(=O)N1CC=C(Br)C(OCC)C1</smiles>

Compound 12: To a solution of alcohol $11(0.54 \mathrm{mg}, 1.81 \mathrm{mmol})$ in dichloromethane $(12 \mathrm{~mL})$ and triethylamine $(2 \mathrm{~mL})$ at $0^{\circ} \mathrm{C}$ were added chlorodiethylphosphate $(0.30 \mathrm{~mL}, 2.06 \mathrm{mmol})$ and a catalytic amount of DMAP. Further chlorodiethylphosphate $(0.10 \mathrm{~mL}, 0.69 \mathrm{mmol})$ was added after $30 \mathrm{~min}$ and the reaction allowed to warm to rt over $2 \mathrm{~h}$ before being partitioned between dichloromethane and brine. The aqueous layer was extracted with further dicloromethane and the combined organic extracts concentrated in vacuo. Purification by column chromatography (50-100\% ether/hexane) afforded 12 as a colourless oil $(0.57 \mathrm{~g}$, $73 \%)$ that crystallised upon storage in the freezer. $R_{f}=0.20$ (75\% ethyl acetate/hexane); $[\alpha]_{D}$ -18.4 $\left(\mathrm{CH}_{2} \mathrm{Cl}_{2}, \mathrm{c}=0.043\right) ;{ }^{1} \mathrm{H}$ NMR $\left(400 \mathrm{MHz}, \mathrm{CDCl}_{3}\right) \delta 1.34$ (two overlapping quartets, $\mathrm{J}=$ $7.0 \mathrm{~Hz}, 6 \mathrm{H}$ in total), $1.38(\mathrm{~s}, 9 \mathrm{H}), 3.59(\mathrm{dd}, J=14.1$ and $3.5 \mathrm{~Hz}, 1 \mathrm{H}), 3.76-4.06(\mathrm{~m}, 3 \mathrm{H}), 4.19$ (two overlapping quintets, $J=7.0 \mathrm{~Hz}, 4 \mathrm{H}$ in total), 4.84-4.90 $(\mathrm{m}, 1 \mathrm{H}), 6.33(\mathrm{t}, J=3.5 \mathrm{~Hz}) ;{ }^{13} \mathrm{C}$ $\operatorname{NMR}\left(100 \mathrm{MHz}, \mathrm{CDCl}_{3}\right) \delta 16.0,16.1,24.5,47.8,50.3,62.1,64.35$ (d, $\left.\mathrm{J}_{\mathrm{C}-\mathrm{P}}=6.9 \mathrm{~Hz}\right), 64.42(\mathrm{~d}$, $\left.J_{\mathrm{C}-\mathrm{P}}=6.9 \mathrm{~Hz}\right), 73.3\left(\mathrm{~d}, J_{\mathrm{C}-\mathrm{P}}=3.4 \mathrm{~Hz}\right), 118.6\left(\mathrm{~d}, J_{\mathrm{C}-\mathrm{P}}=7.6 \mathrm{~Hz}\right), 131.4 ; \mathrm{ms}(\mathrm{ES}) \mathrm{m} / \mathrm{z}(\%) 434$ $\left.\left.\left({ }^{[9} \mathrm{Br}\right] \mathrm{M}^{+}, 89\right), 436\left({ }^{81} \mathrm{Br}\right] \mathrm{M}^{+}, 100\right), 456\left(\left[{ }^{79} \mathrm{Br}_{\mathrm{M}}^{+}+\mathrm{Na}, 71\right), 458\left({ }^{81} \mathrm{Br}\right] \mathrm{M}^{+}+\mathrm{Na}, 70\right) ;$ High resolution ms: expected for $\mathrm{C}_{13} \mathrm{H}_{26} \mathrm{BrNO}_{6} \mathrm{~S} 434.0396$, found 434.0384 .<smiles>C[C@H]1CN(S(=O)(=O)C(C)(C)C)CC=C1Br</smiles>

Compound 13: To a slurry of $\mathrm{CuBr} \cdot \mathrm{SMe}_{2}(0.77 \mathrm{~g}, 3.73 \mathrm{mmol})$ in THF $(6 \mathrm{~mL})$ at $0^{\circ} \mathrm{C}$ was added methylmagnesium chloride $(1.2 \mathrm{~mL}$ of a $3.0 \mathrm{M}$ solution in diethyl ether, $3.60 \mathrm{mmol})$. The resultant yellow mixture was stirred for $30 \mathrm{~min}$ before the temperature of the cooling bath was lowered to $-40^{\circ} \mathrm{C}$ and a solution of $12(0.80 \mathrm{~g}, 1.84 \mathrm{mmol})$ in THF $(10+2 \mathrm{~mL})$ added dropwise. The reaction was maintained at or below $-35^{\circ} \mathrm{C}$ until complete by TLC (ca. $4-5 \mathrm{~h}$ ) then quenched with saturated aqueous ammonium chloride and extracted with ether. The aqueous layer was extracted $(2 \times)$ with further ether and the combined organic phases washed with brine and dried over anhydrous magnesium sulphate. Filtration through Celite and concentration in vacuo afforded 13 a colourless solid $(0.523 \mathrm{~g}, 96 \%)$ which was pure enough to be used in the next step without further purification. An analytical sample was obtained by column chromatography (3:1, ether-hexane). $R_{f}=0.52$ (3:5, ethyl acetate-hexane); $[a]_{D}-9.0$

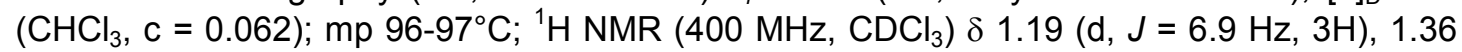
$(\mathrm{s}, 9 \mathrm{H}), 2.59(\mathrm{br} \mathrm{s}, 1 \mathrm{H}), 3.23-3.33(\mathrm{~m}, 1 \mathrm{H}), 3.61(\mathrm{dd}, J=13.1$ and $4.4 \mathrm{~Hz}, 1 \mathrm{H}), 3.89(\mathrm{br} \mathrm{s}, 2 \mathrm{H})$, $6.00(\mathrm{td}, J=2.6$ and $1.2 \mathrm{~Hz}, 1 \mathrm{H}) ;{ }^{13} \mathrm{C}$ NMR $\left(100 \mathrm{MHz}, \mathrm{CDCl}_{3}\right) \delta 17.9,24.7,38.5,48.0,51.4$, $61.8,125.1,127.0$; ms (ES) $\left.\mathrm{m} / \mathrm{z}(\%) 319{ }^{[81]} \mathrm{BrM}^{+}+\mathrm{Na}, 100\right), 317\left({ }^{[79]} \mathrm{BrM}^{+}+\mathrm{Na}, 89\right)$; High resolution ms: expected for $\mathrm{C}_{10} \mathrm{H}_{19} \mathrm{BrNO}_{2} \mathrm{~S} 296.0314$, found 296.0312 .

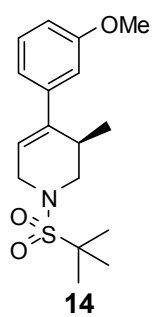


Compound 14: To a solution of (-)-(S)-13 $(0.523 \mathrm{~g}, 1.76 \mathrm{mmol})$ in absolute ethanol-toluene $(1: 1,40 \mathrm{~mL})$ were added 3-methoxybenzeneboronic acid (308 mg, $2.03 \mathrm{mmol}), \mathrm{Pd}\left(\mathrm{Ph}_{3} \mathrm{P}\right)_{4}(96$ $\mathrm{mg}, 83 \mu \mathrm{mol}, 4.7 \mathrm{~mol} \%)$ and $1 \mathrm{M}$ aqueous sodium carbonate $(7.0 \mathrm{~mL})$ and the reaction mixture heated at $80^{\circ} \mathrm{C}$ for $45 \mathrm{~min}$. Most of the ethanol was then removed by rotary evaporation and the resultant red-brown mixture partitioned between diethyl ether and water. The organic phase was then washed with brine, dried over magnesium sulfate and concentrated in vacuo. Column chromatography ( $15 \%$ ethyl acetate/hexane) afforded 14 as a colourless oil $\left(0.58 \mathrm{~g}, 97 \%\right.$ for 2 steps) that crystallised upon storage in the freezer. $R_{f}=0.49$ (3:5, ethyl acetate-hexane); $[\alpha]_{\mathrm{D}}-62.1\left(\mathrm{CHCl}_{3}, \mathrm{c}=0.078\right), \mathrm{mp} 96-97^{\circ} \mathrm{C}, \mathrm{IR}$ (neat) $3417(\mathrm{w})$, 2970, 2930, 1598, 1580, 1489, 1463, 1315(s), 1150, 1124, 1027, $715 \mathrm{~cm}^{-1} ;{ }^{1} \mathrm{H}$ NMR (270 $\left.\mathrm{MHz}, \mathrm{CDCl}_{3}\right) \delta 1.04(\mathrm{~d}, J=6.9 \mathrm{~Hz}, 3 \mathrm{H}), 1.41(\mathrm{~s}, 9 \mathrm{H}), 2.87(\mathrm{br} \mathrm{s}, 1 \mathrm{H}), 3.47-3.58(\mathrm{~m}, 1 \mathrm{H}), 3.80$ (s, 3H), $4.08(\mathrm{br} \mathrm{q}, 2 \mathrm{H}), 5.82(\mathrm{ddd}, J=3.2,3.2$ and $0.8 \mathrm{~Hz}, 1 \mathrm{H}), 6.80-6.84(\mathrm{~m}, 2 \mathrm{H}), 6.88(\mathrm{dt}, J$ $=7.9$ and $1.2 \mathrm{~Hz}, 1 \mathrm{H}), 7.23(\mathrm{td}, J=7.4$ and $1.2 \mathrm{~Hz}, 1 \mathrm{H}) ;{ }^{13} \mathrm{C} \mathrm{NMR}\left(100 \mathrm{MHz}, \mathrm{CDCl}_{3}\right) \delta 17.8$, 24.8, 32.2, 46.8, 50.9, 55.2, 61.8, 112.0, 112.5, 118.5, 120.6, 129.3, 141.54, 141.60, 159.6, ms (ES+) m/z (\%) $346\left(\mathrm{M}^{+}+\mathrm{Na}, 100\right), 324\left(\mathrm{MH}^{+}, 54\right), 226$ (22); High resolution ms: expected for $\mathrm{C}_{17} \mathrm{H}_{26} \mathrm{NO}_{3} \mathrm{~S} 324.1628$, found 324.1613.

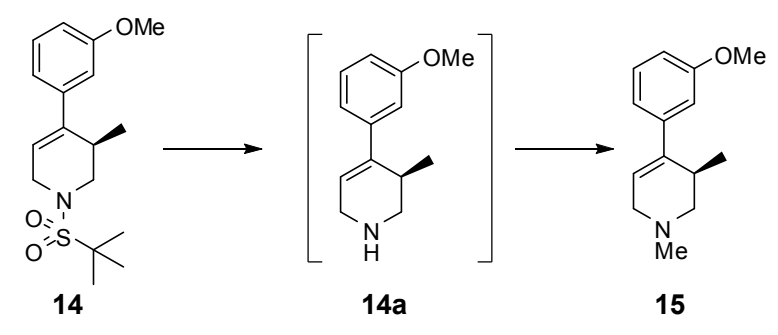

Compound 15: To a solution of $14(0.56 \mathrm{~g}, 1.73 \mathrm{mmol})$ in dichloromethane $(15 \mathrm{~mL})$ containing anisole $(1.0 \mathrm{~mL})$ at $0^{\circ} \mathrm{C}$ was added a solution of triflic acid in dichloromethane $(0.2 \mathrm{M}, 15 \mathrm{~mL}, 7.50 \mathrm{mmol})$. When complete by TLC (ca. $30 \mathrm{~min}$ ) the reaction was quenched by the dropwise addition of $2.0 \mathrm{M} \mathrm{NaOH}(10 \mathrm{~mL})$ and the reaction mixture extracted with dichloromethane $(3 \times)$. The organic phase was dried over potassium carbonate and concentrated in vacuo. Column chromatography (1:1 hexane-dichloromethane then 90:10:1, $\mathrm{CH}_{2} \mathrm{Cl}_{2}-\mathrm{MeOH}$-aq. $\left.\mathrm{NH}_{3}\right)$ afforded $14 \mathrm{a}(0.31 \mathrm{~g}, 86 \%)$ as a colourless oil. $\mathrm{R}_{f}=0.15$ (90:10:1, $\mathrm{CH}_{2} \mathrm{Cl}_{2}-\mathrm{MeOH}$-aq. $\left.\mathrm{NH}_{3}\right) ;[\alpha]_{\mathrm{D}}-91.1\left(\mathrm{CHCl}_{3}, \mathrm{c}=0.019\right)$, IR (neat) 3392(br), 2959, 2834, 1598, $1576,1558,1489,1457,1418,1286,1265,1205,1170,1039,772 \mathrm{~cm}^{-1} ;{ }^{1} \mathrm{H}$ NMR $(400 \mathrm{MHz}$, $\left.\mathrm{CDCl}_{3}\right) \delta 0.97(\mathrm{~d}, J=7.0 \mathrm{~Hz}, 3 \mathrm{H}), 2.30(\mathrm{br} \mathrm{s}, 1 \mathrm{H}), 2.73(\mathrm{br} \mathrm{s}, 1 \mathrm{H}), 2.83(\mathrm{dd}, J=12.5$ and 3.9 $\mathrm{Hz}, 1 \mathrm{H}$ ), $3.13(\mathrm{dd}, J=12.5$ and $4.7 \mathrm{~Hz}, 1 \mathrm{H}), 3.46(\mathrm{dd}, J=5.5$ and $3.3 \mathrm{~Hz}, 2 \mathrm{H}), 3.80(\mathrm{~s}, 3 \mathrm{H})$, 5.92 (ddd, $J=3.4,3.4$ and $0.9 \mathrm{~Hz}, 1 \mathrm{H}$ ), $6.78(\mathrm{dd}, J=8.2$ and $2.2 \mathrm{~Hz}, 1 \mathrm{H}), 6.84(\mathrm{t}, J=2.2 \mathrm{~Hz}$, $1 \mathrm{H}), 7.23(\mathrm{t}, J=8.2 \mathrm{~Hz}, 1 \mathrm{H}) ;{ }^{13} \mathrm{C}$ NMR $\left(100 \mathrm{MHz}, \mathrm{CDCl}_{3}\right) \delta 18.3,30.6,45.8,50.5,55.2,111.9$, 112.0, 118.5, 124.4, 129.2, 141.5, 142.8, 159.5; ms (ES+) $\mathrm{m} / \mathrm{z}(\%) 204\left(\mathrm{MH}^{+}, 100\right), 175(50)$; High resolution ms: expected for $\mathrm{C}_{13} \mathrm{H}_{18} \mathrm{NO} 204.1383$, found 204.1374 .

To $14 \mathrm{a}(0.26 \mathrm{~g}, 1.28 \mathrm{mmol})$ in acetonitrile $(10 \mathrm{~mL})$ were added sodium cyanoborohydride (225 $\mathrm{mg}, 3.57 \mathrm{mmol}$ ) and $37 \%$ aqueous formaldehyde $(0.23 \mathrm{~mL}, 2.83 \mathrm{mmol})$. After $30 \mathrm{~min}$ the reaction was quenched with acetic acid $(0.50 \mathrm{~mL})$ then basified with $2.0 \mathrm{M} \mathrm{NaOH}$. The reaction mixture was extracted with dichloromethane $(3 \times)$ and the combined organic phases dried over anhydrous potassium carbonate and concentrated in vacuo. Column chromatography (100:0:0-90:10:1, ethyl acetate-MeOH-aq. $\left.\mathrm{NH}_{3}\right)$ afforded $15(0.24 \mathrm{~g}, 87 \%)$ was a yellow oil: $\mathrm{R}_{f}=0.18\left(90: 10: 1, \mathrm{CH}_{2} \mathrm{Cl}_{2}-\mathrm{MeOH}\right.$-aq. $\left.\mathrm{NH}_{3}\right)$; $[\alpha]_{\mathrm{D}}-88.4\left(\mathrm{CHCl}_{3}, \mathrm{c}=0.073\right)$; ${ }^{1} \mathrm{H}$ NMR $\left(400 \mathrm{MHz}, \mathrm{CDCl}_{3}\right) \delta 0.99(\mathrm{~d}, J=7.0 \mathrm{~Hz}, 3 \mathrm{H}), 2.37(\mathrm{~s}, 3 \mathrm{H}), 2.38$ (dd, $J=11.1$ and 5.1 $\mathrm{Hz}, 1 \mathrm{H}), 2.68(\mathrm{dd}, J=11.1$ and $4.9 \mathrm{~Hz}, 1 \mathrm{H}), 2.89$ (br s, 1H), 3.03 (dddd, $J=42.1,16.6,2.8$ and $2.8 \mathrm{~Hz}, 2 \mathrm{H}$ ), $3.80(\mathrm{~s}, 3 \mathrm{H}), 5.84(\mathrm{ddd}, J=3.5,3.5$ and $1.2 \mathrm{~Hz}, 1 \mathrm{H}), 6.78$ (ddd, $J=7.8,2.7$ and $0.7 \mathrm{~Hz}, 1 \mathrm{H}), 6.85(\mathrm{t}, J=2.1 \mathrm{~Hz}, 1 \mathrm{H}), 6.89(\mathrm{ddd}, J=7.5,1.6$ and $1.6 \mathrm{~Hz}, 1 \mathrm{H}), 7.22(\mathrm{t}, J=$ $8.0 \mathrm{~Hz}, 1 \mathrm{H}) ;{ }^{13} \mathrm{C}$ NMR $\left(100 \mathrm{MHz}, \mathrm{CDCl}_{3}\right) \delta 18.7,32.2,45.9,55.2,55.4,60.2,112.0,112.1$, 118.7, 122.6, 129.1, 141.0, 142.5, 159.5; ms (ES+) $\mathrm{m} / \mathrm{z}(\%) 218\left(\mathrm{MH}^{+}, 100\right)$; High resolution ms: expected for $\mathrm{C}_{14} \mathrm{H}_{20} \mathrm{NO} 218.1539$, found 218.1533. 


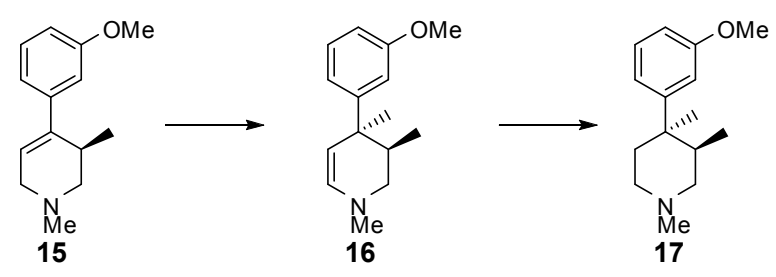

Compound 17: Prepared according to the literature procedure reported for the isopropyl analogue. $^{4 \mathrm{~b}} \mathrm{R}_{f}=0.1\left(90: 10: 1, \mathrm{CH}_{2} \mathrm{Cl}_{2}-\mathrm{MeOH}\right.$-aq. $\left.\mathrm{NH}_{3}\right) ;{ }^{1} \mathrm{H} \mathrm{NMR}\left(400 \mathrm{MHz}, \mathrm{CDCl}_{3}\right) \delta 0.78$ (d, $J=7.0 \mathrm{~Hz}, 3 \mathrm{H}), 1.30(\mathrm{~s}, 3 \mathrm{H}), 1.57-1.61(\mathrm{~m}, 1 \mathrm{H}), 1.95-2.02(\mathrm{~m}, 1 \mathrm{H}), 2.27(\mathrm{~s}, 3 \mathrm{H}), 2.28-2.33$ $(\mathrm{m}, 1 \mathrm{H}), 2.52(\mathrm{~d}, J=3.1 \mathrm{~Hz}, 2 \mathrm{H}), 2.77-2.81(\mathrm{~m}, 1 \mathrm{H}), 3.79(\mathrm{~s}, 3 \mathrm{H}), 6.70$ (ddd, $J=8.2,2.7$ and $0.8 \mathrm{~Hz}, 1 \mathrm{H}$ ), 6.85 (t, $J=2.2 \mathrm{~Hz}, 1 \mathrm{H}), 6.88$ (ddd, $J=7.8,2.0$ and $0.8 \mathrm{~Hz}, 1 \mathrm{H}), 7.22(\mathrm{t}, J=8.0$ $\mathrm{Hz}, 1 \mathrm{H}) ;{ }^{13} \mathrm{C}$ NMR $\left(100 \mathrm{MHz} \mathrm{CDCl}_{3}\right) \delta 16.5,27.6,30.6,38.0,38.7,46.7,52.2,55.1,58.5$, 109.6, 112.4, 118.2, 128.9, 151.2, 159.4; ms (ES) $\mathrm{m} / \mathrm{z}(\%) 234\left(\mathrm{MH}^{+}, 100\right)$; High resolution ms: expected for $\mathrm{C}_{14} \mathrm{H}_{24} \mathrm{NO} 234.1855$, found 234.1845.

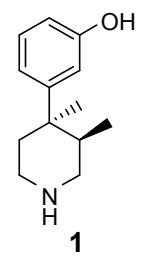

Compound 1: Prepared according to the reported procedure, ${ }^{4 a}$ NMR data was in accordance with that previously reported. ${ }^{4 \mathrm{~b}}[\alpha]_{\mathrm{D}}+103(\mathrm{MeOH}, \mathrm{c}=0.023)$; High resolution ms: expected for $\mathrm{C}_{13} \mathrm{H}_{20} \mathrm{NO} 206.1539$, found 206.1530. HPLC: $t_{\mathrm{R}}$ (minor) $=14.5 \mathrm{~min}, t_{\mathrm{R}}$ (major) $=15.8 \mathrm{~min}$ detected at $274 \mathrm{~nm}, 80: 20$ (MeOH-0.1\% ammonium trifluoroacetate in $\mathrm{MeOH}$ ), $1 \mathrm{~mL} / \mathrm{min}$. 